\title{
Atomic nuclei decay modes by spontaneous emission of heavy ions
}

\author{
D. N. Poenaru and M. Ivaşcu \\ Central Institute of Physics, 76900 Bucharest, Romania
}

A. Săndulescu

Joint Institute for Nuclear Research, 141980 Dubna, USSR

\author{
Walter Greiner \\ Johann-Wolfgang-Goethe Universität, D-6000 Frankfurt am Main, Federal Republic of Germany
}

(Received 5 November 1984)

\begin{abstract}
The great majority of the known nuclides with $Z>40$, including the so-called stable nuclides, are metastable with respect to several modes of spontaneous superasymmetric splitting. A model extended from the fission theory of alpha decay allows one to estimate the lifetimes and the branching ratios relative to the alpha decay for these natural radioactivities. From a huge amount of systematic calculations it is concluded that the process should proceed with maximum intensity in the translead nuclei, where the minimum lifetime is obtained from parent-emitted heavy ion combinations leading to a magic $\left({ }^{208} \mathrm{~Pb}\right)$ or almost magic daughter nucleus. More than 140 nuclides with atomic number smaller than 25 are possible candidates to be emitted from heavy nuclei, with half-lives in the range of $10^{10}-10^{30} \mathrm{~s}:{ }^{5} \mathrm{He},{ }^{8-10} \mathrm{Be},{ }^{11,12} \mathrm{~B},{ }^{12-16} \mathrm{C},{ }^{13-17} \mathrm{~N},{ }^{15-22} \mathrm{O},{ }^{18-23} \mathrm{~F},{ }^{20-26} \mathrm{Ne},{ }^{23-28} \mathrm{Na},{ }^{23-30} \mathrm{Mg}$,

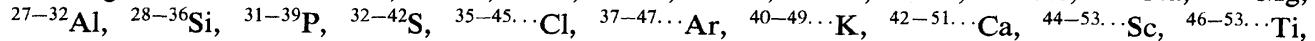

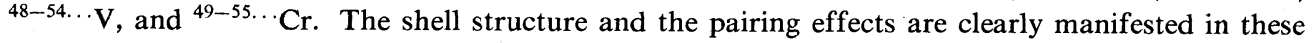
new decay modes.
\end{abstract}

\section{INTRODUCTION}

There are many kinds of radioactivities discovered so far. ${ }^{1,2}$ In a number of previous papers ${ }^{2-8}$ we have predicted new related phenomena, showing that a great variety of heavy ions (HI's) with mass number $A_{2}>4$ and atomic number $Z_{2} \geq 2$ could be emitted from heavy nuclides $(A, Z)$ leading to a daughter $\left(A_{1}, Z_{1}\right)$. Some of the predictions ${ }^{3}$ concerning these "unexpected" have been recently mentioned. ${ }^{10}$

The spontaneous emission of ${ }^{14} \mathrm{C}$ from ${ }^{223} \mathrm{Ra}$, experimentally discovered ${ }^{11}$ at the beginning of 1984 and already confirmed, ${ }^{12,9}$ is the first evidence of one of the new decay modes.

The asymmetry of a typical fission fragment mass distribution curve ${ }^{13}$ was explained ${ }^{14-17}$ as determined by the shell effects. $^{18,19}$ We noticed ${ }^{3,4}$ the gap between $A_{2}=4$ and $A_{2}=60-70$ and we found in this region the new phenomena, assuming the production mechanism to be either an emission of a preformed cluster or a superasymmetric fission process. Several methods have been used.

By means of the fragmentation theory, ${ }^{20}$ allowing to calculate the fission fragment mass distribution, ${ }^{21}$ a new peak $\left({ }^{48} \mathrm{Ca}\right)$ for the ${ }^{252}$ No fission ${ }^{22,3}$ and a shoulder ${ }^{23}$ for the ${ }^{238} \mathrm{U}$ fission has been found. ${ }^{22,3}$ Penetrability calculations ${ }^{3}$ showed that eight even-even $\mathrm{HI}$ 's $-{ }^{14} \mathrm{C},{ }^{24} \mathrm{Ne},{ }^{28} \mathrm{Mg}$, ${ }^{32,34} \mathrm{Si},{ }^{46} \mathrm{Ar}$, and ${ }^{48,50} \mathrm{Ca}$-could be preferentially emitted from 16 nuclides, and isotopes of $\mathrm{Ra}, \mathrm{Th}, \mathrm{U}, \ldots$ up to No.

However, the penetrability is not a measurable quantity. Our program allowing to estimate the half-lives ${ }^{4}$ was based on the fission mechanism. A numerical superasymmetric fission model (NSAFM) was derived by extend- ing $^{24-26}$ three variants of the liquid drop model [LDM, ${ }^{27}$ finite range of nuclear forces model (FRNFM), 28 and Yukawa-plus-exponential model (Y + EM) (Ref. 29)] to the systems with charge asymmetry different from the mass asymmetry and by using a phenomenological shell correction. The model was tested ${ }^{30,31,3,4}$ for $\alpha$ decay, the half-lives being computed with the Wentzel-KramersBrillouin (WKB) method successfully used ${ }^{32}$ in fission. Another model describing in a unified way the statistics of light particle emission (from highly excited states above the barrier) and the fission was developed in Refs. 33 and 34 and was recently tested. ${ }^{35}$ Unlike this model we are concerned with ground or low excited states below the barrier, where quantum-mechanical tunneling and shell effects are very important.

The NSAFM involving manifold numerical quadratures is too slow to be used for a systematic search of new decay modes, where one has more than $10^{5}$ combinations of parent-emitted HI's. An analytical relationship [analytical superasymmetric fission model (ASAFM)] was derived $^{4}$ and extended ${ }^{5}$ to account for angular momentum and small excitation energy effects. We have seen ${ }^{4,5}$ that all $\sim 2000$ nuclides of known masses ${ }^{36}$ are stable for the emission of ${ }^{2,3} \mathrm{H},{ }^{3,6-9} \mathrm{He},{ }^{4} \mathrm{Li},{ }^{7} \mathrm{~B}$, and ${ }^{9} \mathrm{C}$ but ${ }^{8} \mathrm{Be},{ }^{12} \mathrm{C}$, ${ }^{16} \mathrm{O}$, etc., are good candidates. The simple rule of minimum lifetime for the ${ }^{208} \mathrm{~Pb}$ daughter obtained for the ${ }^{5} \mathrm{He}$ radioactivity ${ }^{5}$ was verified when other candidates with an increased $Z_{2}(4-10)$ were considered. ${ }^{6-8}$

In this paper, we report a more comprehensive list ${ }^{2}$ $\left(Z_{2}=2-24\right)$ of the decay modes and an improved accuracy which is made possible by the use of the new mass tables $^{37}$ and the introduction of some shell effects in the 
zero point vibration energy $E_{v}$, giving a better fit with experimental data for the $380 \alpha$ emitters and reproducing the ${ }^{14} \mathrm{C}$ radioactivity of the ${ }^{223} \mathrm{Ra}$ experimental point. In this way, we expect to estimate better the lifetimes for the new decay modes, especially in the neighborhood of the magic neutron and proton numbers of the daughter.

\section{CALCULATION OF THE BARRIER AND THE LIFETIME}

We are looking for metastable states, ${ }^{4}$ for which both the released energy

$$
Q=M(A, Z)-\left[M\left(A_{1}, Z_{1}\right)+M\left(A_{2}, Z_{2}\right)\right]
$$

and the barrier height are positive. The mass excess $M(A, Z)$ is taken from the tables. ${ }^{37}$

Two parametrizations for nuclear shapes are adopted: intersected spheres, ${ }^{30}$ or a spheroid and a small sphere. ${ }^{31}$ In the first case, the separation distance between centers is varied from $R_{i}=R_{0}-R_{2}$ to infinity, where $R_{0}=r_{0} A^{1 / 3}$, $R_{j}=r_{0} A_{j}^{1 / 3}(j=1,2)$. The radius constant $r_{0}$ takes different values when the liquid drop part $E_{\mathrm{LD}}$ in the equation of the deformation energy

$$
E(R)=E_{\mathrm{LD}}(R)+\delta E(R)
$$

is computed ${ }^{38}$ by using LDM, FRNFM, or Y + EM. By choosing the origin of the potential energy at $R=\infty$ and a suitable ${ }^{30}$ shell correction term $\delta E$, one has $E\left(R_{i}\right)=Q$.

The half-life of a metastable system is given ${ }^{32}$ by

$$
T=\frac{\hbar \ln 2}{\Gamma}=\frac{\ln 2}{v P},
$$

where $\Gamma$ is the partial disintegration width for a particular mode and

$$
v=\frac{\omega}{2 \pi}=\frac{2 E_{v}}{h}
$$

represents the number of assaults on the barrier per second. According to the WKB theory, the probability per unit time of penetration through the barrier

$$
P=[1+\exp (K)]^{-1} \simeq \exp (-K)
$$

where the action integral

$$
K=\frac{2}{\hbar} \int_{R_{a}}^{R_{b}}\left\{2 \mu\left[E(R)-Q^{\prime}\right]\right\}^{1 / 2} d R,
$$

the mass parameter $\mu$ is replaced by the reduced mass $\mu=m_{n} A_{1} A_{2} / A, m_{n}$ is the nucleon mass, $R_{a}$ and $R_{b}$ are given by $E\left(R_{a}\right)=E\left(R_{b}\right)=Q^{\prime}$, and

$$
Q^{\prime}=Q+E_{v} \text {. }
$$

By comparing

$$
\Gamma=\frac{E_{v} P}{\pi}
$$

with $\Gamma=2 \gamma^{2} P^{\prime}$ used in the $R$-matrix theory of $\alpha$ decay, it seems that the role of the reduced width $\gamma^{2}$, proportional to the cluster preformation probability, is played by $E_{v}$. This is not true. The penetrability $P^{\prime}$ is not dependent on $\gamma^{2}$; it is calculated for $R>R_{t}=R_{1}+R_{2}$, where electrostatic forces dominate, by considering a point in a potential. In fission theory, ${ }^{32} P$ is function of $E_{v}, E(R)$ is the interaction energy of two extended objects, and the contribution of the strong interaction in the overlapping region of the fragments $\left(R_{i}<R<R_{t}\right)$ increases with $A_{2}$. The variation of $E_{v}$ with the neutron number $N_{1}$ for $\alpha$ decay or with $A_{2}$ from cluster to cluster is much smaller than that of $\gamma^{2}$. This stability of $E_{v}$ is a useful property for lifetime predictions. The argument of some experimenters that they have found a good $P^{\prime} / P_{\alpha}^{\prime}$ ratio, does not imply that the branching ratio

$$
\frac{T_{\alpha}}{T}=\frac{\Gamma}{\Gamma_{\alpha}}=\frac{\gamma^{2}}{\gamma_{\alpha}^{2}} \frac{P^{\prime}}{P_{\alpha}^{\prime}}=\frac{E_{v}}{E_{v \alpha}} \frac{P}{P_{\alpha}}
$$

will also be good, as long as for clusters heavier than $\alpha$ it is not known how to calculate or to measure $\gamma^{2}$.

In the framework of NSAFM, $E_{v}$ was found from a fit with experimental data. The best overall agreement with data, ranging over 24 orders of magnitude, for $58 \alpha$ emitters has been obtained with $E_{v}=0.37$ and $0.3 \mathrm{MeV}$ when $E_{\mathrm{LD}}$ was calculated by LDM and Y + EM, respectively. A time-dependent Hartree-Fock (TDHF) computation $^{39}$ for the system $\alpha+{ }^{208} \mathrm{~Pb}$ led to $E_{v} \simeq 0.4 \mathrm{MeV}$. For the ${ }^{14} \mathrm{C}$ radioactivity of ${ }^{223} \mathrm{Ra}$, one needs $0.025 \mathrm{MeV}$ $(\mathrm{Y}+\mathrm{EM})$.

The analytical relationship (ASAFM) for the lifetime was derived ${ }^{4,5}$ by approximating the potential barrier $E(R)$ in the overlapping region by a second-order polynomial in $R$. Sometimes, from the spin and parity conservation $^{5}$ one has a nonzero angular momentum $l \hbar$. The centrifugal barrier gives an additional delay. The excitation energy, supplied for example by the $\beta$ decay of a precursor, acts in the opposite direction.

If we express the time in seconds, the energies in $\mathrm{MeV}$, and the lengths in $\mathrm{fm}$, the decimal logarithm of the halflife is given by

$$
\begin{aligned}
& \log T=0.43429\left(K_{o v}+K_{s}\right)-\log E_{v}-20.8436 \\
& K_{o v}=0.2196\left(E_{b}^{0} A_{1} A_{2} / A\right)^{1 / 2}\left\{\left(b^{2}-a^{2}\right)^{1 / 2}-\frac{a^{2}}{b} \ln \left[\frac{b+\left(b^{2}-a^{2}\right)^{1 / 2}}{a}\right]\right\}, \\
& K_{s}=0.4392\left[\frac{Q^{\prime} A_{1} A_{2}}{A}\right]^{1 / 2} R_{b} \mathscr{T}_{m c}, \\
& E_{i}=E_{c}+E_{l} ; \quad E_{c}=\frac{1.44 Z_{1} Z_{2}}{R_{t}} ; E_{l}=\frac{20.735 l(l+1) A}{R_{t}^{2} A_{1} A_{2}}
\end{aligned}
$$




$$
\begin{aligned}
& R_{b}=\frac{R_{t} E_{c}}{Q^{\prime}}\left[\frac{1}{2}+\left[\frac{1}{4}+\frac{Q^{\prime} E_{l}}{E_{c}^{2}}\right]^{1 / 2}\right] ; r=\frac{R_{t}}{R_{b}}, \\
& Q^{\prime}=Q+E_{v}+E^{*} ; a=b\left[\frac{Q^{\prime}-Q}{E_{b}^{0}}\right]^{1 / 2} ; b=R_{t}-R_{i}, \\
& \mathscr{T}_{m c}=(c+m-1)^{1 / 2}-[r(c-r)+m]^{1 / 2}+\frac{c}{2}\left[\arcsin \frac{c-2 r}{\left(c^{2}+4 m\right)^{1 / 2}}-\arcsin \frac{c-2}{\left(c^{2}+4 m\right)^{1 / 2}}\right] \\
& \quad+\sqrt{m} \ln \left\{\frac{2 \sqrt{m}[r(c-r)+m]^{1 / 2}+c r+2 m}{r\left[2 \sqrt{m}(c+m-1)^{1 / 2}+c+2 m\right]}\right\} \\
& m=\frac{r^{2} E_{l}}{Q^{\prime}} ; c=\frac{r E_{c}}{Q^{\prime}} ; r_{0}=1.2249 \mathrm{fm} ;
\end{aligned}
$$

where $E^{*}$ is the fraction of the excitation energy, concentrated in the collective mode leading to separation.

At the beginning ${ }^{5,6}$ the zero point vibration energy was obtained by a fit with a selected set of experimental data ${ }^{40}$ $T_{i}^{\exp }$ on $376 \alpha$ emitters. Now there are $380 \alpha$-decaying nuclides ${ }^{41}$ and as is shown by curve (a) from Fig. 1, for $E_{v}=0.51 \mathrm{MeV}$, the standard deviation of $\log T$,

$$
\sigma=\left[\frac{1}{379} \sum_{i=1}^{380}\left(\log T_{i}-\log T_{i}^{\exp }\right)^{2}\right]^{1 / 2},
$$

takes the minimum value $\sigma=0.96$.

When the measurement for ${ }^{14} \mathrm{C}$ radioactivity was available, ${ }^{11}$ we saw that our predictions made with $E_{v}=0.51$ $\mathrm{MeV}$ had been by three orders of magnitude too pessimistic. Agreement has been obtained ${ }^{7,8}$ by using the law $E_{v}=0.1275 A_{2}$.

Using a semiempirical formula ${ }^{4,42}$ for $\alpha$ decay, in which the shell effects have been taken into account, one gets $\sigma=0.41$. The variation of $E_{v}$ calculated to reproduce exactly the lifetime for each $\alpha$ emitter, suggests a simple way to introduce shell effects in $E_{v}$ by making it proportional to $Q$. Curve (b) of Fig. 1 shows the optimum value $0.095 Q$ for which $\sigma=0.73$. A convenient law was found ${ }^{2}$ to be

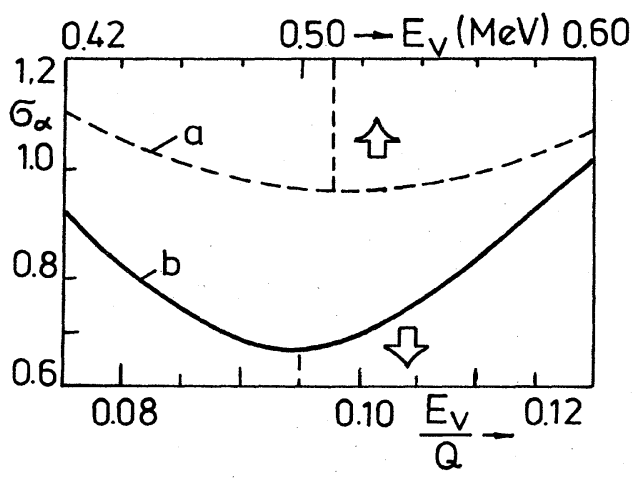

FIG. 1. Choosing the zero point vibration energy $E_{v}$ by minimization of the standard deviation of $\log T$ values for $380 \alpha$ emitters. $E_{v}=$ constant (a) and $E_{v}$ proportional with $Q_{\alpha}$, when $Q_{\alpha}>0$ (b).

$$
\begin{aligned}
& E_{v}=Q\left[0.056+0.039 \exp \left[\frac{4-A_{2}}{2.5}\right)\right] \text {; } \\
& Q>0 ; A_{2} \geq 4 \text {. }
\end{aligned}
$$

One has $E_{v}=0.095 Q$ for $\alpha$ decay and $E_{v}=31.8$ $\times 0.056 \simeq 1.77 \mathrm{MeV}$ for the ${ }^{14} \mathrm{C}$ radioactivity of ${ }^{223} \mathrm{Ra}$.

This equation will be used below. We need further experimental data in order to check and improve the accuracy of the predictions. One can get only rough estimations based on this simple model, but the most interesting results obtained in this way could be studied experimentally or with more refined theoretical models.

From Fig. 2, one can see that a small angular momen-

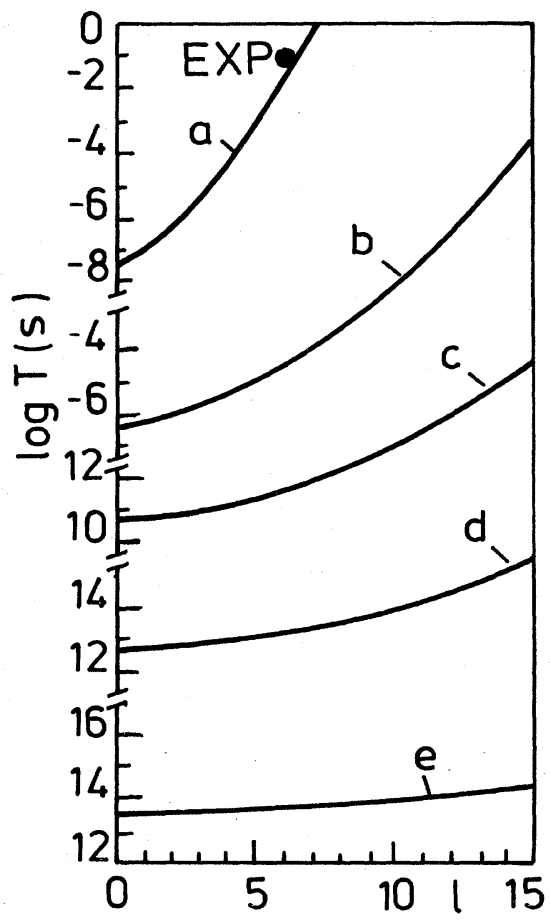

FIG. 2. The centrifugal barrier effect on the lifetime for emission of various charged particles: (a) protons from ${ }^{151} \mathrm{Lu}$ $\left(E_{v}=0.51 \mathrm{MeV}\right.$ ), (b) $\alpha$ from ${ }^{212} \mathrm{Po}$, (c) ${ }^{8} \mathrm{Be}$ from ${ }^{216} \mathrm{Rn}$, (d) ${ }^{14} \mathrm{C}$ from ${ }^{222} \mathrm{Ra}$, and (e) ${ }^{48} \mathrm{Ca}$ from ${ }^{256}$ No. 
tum, up to five units of $\hbar$, makes an important contribution to the lifetime only for light emitted particles (especially for protons). For heavier ions, one can neglect small angular momentum effects; hence in the following we shall not consider the spin and parity conservation.

\section{LIFETIME PREDICTIONS}

In a systematic search for new decay modes, the first step consists in finding the regions of the nuclear table where the condition of metastability is fulfilled. Up to now we have done calculations in which all isotopes of the elements with $Z_{2}<25$ are supposed to be possible candidates of emitted HI's from all nuclides with $A>2 A_{2}$ and $Z>2 Z_{2}$. Only the 2200 nuclides tabulated by Wapstra and Audi were usually considered, but sometimes we extended the region by using a computer program for mass extrapolations ${ }^{43}$ and some of the 1975 mass predictions. ${ }^{44}$

Like the diproton, dineutron, ${ }^{1}$ and trineutron, ${ }^{5} \mathrm{He}$ and ${ }^{8} \mathrm{Be}$ are unstable, but one can assume a mechanism in which the components are leaving the parent nucleus together and after tunneling through the potential barrier they are moving apart.

A part of the nuclear chart, with $Z \geq 60$ and $N \geq 80$, can be seen in Fig. 3, where the contour plots of $Q$ values for ${ }^{14} \mathrm{C}$ emission are drawn. For a given mass number, $A=Z+N$, there is a smooth (liquid-drop model) trend of increase from the neutron rich side toward the neutron deficient side, like for $\alpha$ decay $Q$ values. The reverse is true for very neutron rich emitted HI's like ${ }^{8,9} \mathrm{He}$. The shell effects for the daughter magic numbers $Z_{1}=82, N_{1}=82$ and 126 are also very clearly seen, pushing down the $Q$ value for ${ }^{14} \mathrm{C}$ emission from ${ }_{88}^{222} \mathrm{Ra}(33.05 \mathrm{MeV})$ and from ${ }_{89}^{222} \mathrm{Ac}(33.08 \mathrm{MeV})$.

By comparing Fig. 3 with a similar one for $\alpha$ decay, one can see that the condition of metastability for $\mathrm{HI}$ emission is fulfilled for a larger region of the nuclear chart. From this point of view, these exotic radioactivities are more general phenomena, but their intensities are weaker (the lifetimes larger) than that of $\alpha$ decay.

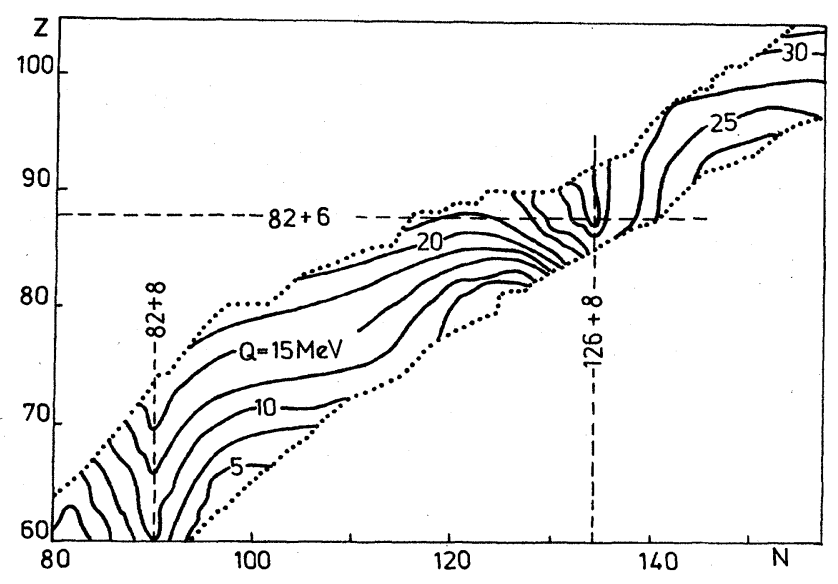

FIG. 3. Contour plots of $Q$ values for ${ }^{14} \mathrm{C}$ emission from various nuclides with $Z \geq 60$ and $N \geq 80$ having masses tabulated by Wapstra and Bos (Ref. 36).
The very strong shell effect at the double magic daughter ${ }^{208} \mathrm{~Pb}$ in Fig. 3, which had been seen in the asymmetric fission mass distribution calculated with the fragmentation theory, ${ }^{22,3}$ was also observed for all other emitted HI's. Hence, we can resume in Fig. 4 the $Q$ values for the $\mathrm{HI}$ emission in which the daughter is ${ }^{208} \mathrm{~Pb}$. The released energy increases with $Z_{2}$ and for $Z_{2}>16$, one has $Q>100 \mathrm{MeV}$. This energy is shared between the emitted particle and the daughter. One can assume that like for $\alpha$ decay the kinetic energy of the $\mathrm{HI}$ is given by $E_{k}=Q A_{1} / A$ and the recoil energy of the daughter is $Q-E$. But it can happen that, for heavier emitted particles, like in fission, a part of this energy is lost on exciting or deforming the fragments.

The corresponding predicted lifetimes are plotted in Fig. 5, displaying the best values expected for almost all new decay modes. To guide the eye, various isotopes of a given emitted element are joined with a heavy line if $Z_{2}$ is even, or with a dotted line if $Z_{2}$ is odd. The specified mass numbers of some emitted HI's are used as reference points. For a practical reason, $Z_{2}$ is restricted to values smaller than 26 (parents with $Z<108$ ), but of course we can continue even for the superheavy nuclei.

Almost all nuclei are metastable with respect to several decay modes, but if the lifetimes are very long, they are stable from the practical point of view. If the age of the universe is of the order of $10^{10} \mathrm{yr}$ (that is $10^{17.5} \mathrm{~s}$ ), then the processes with a half-life much shorter than that explain why some nuclides could not be found in nature. Of course, one has to consider all the competing decay modes, the most important determining the minimum partial half-life. The available experimental techniques allow to measure very slow processes $\left(T \sim 10^{22} \mathrm{yr}\right)$, as for example the spontaneous fission of some actinides. Con-

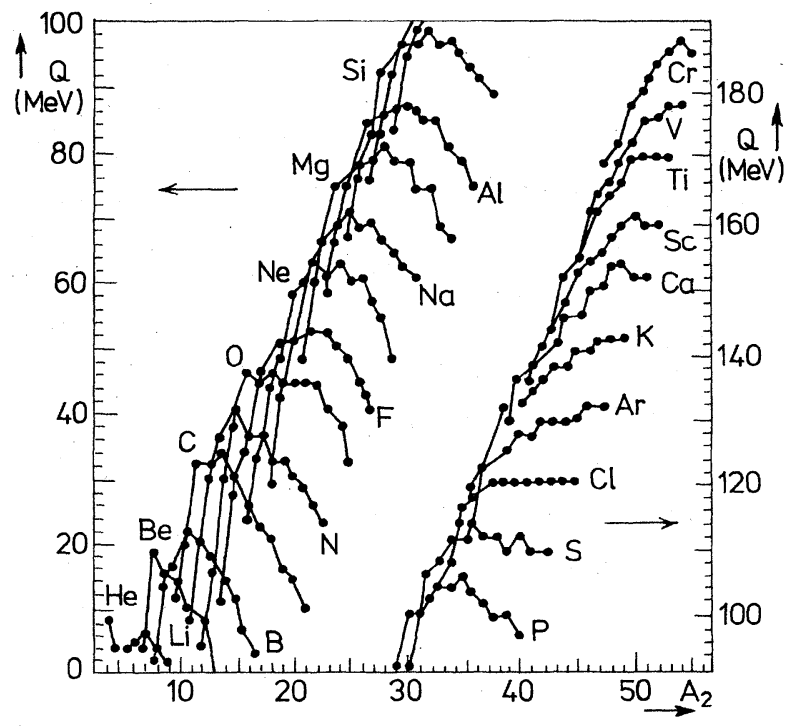

FIG. 4. The released energy for the emission of various isotopes of the specified elements from the parents leading to the double magic ${ }^{208} \mathrm{~Pb}$ daughter nucleus. 


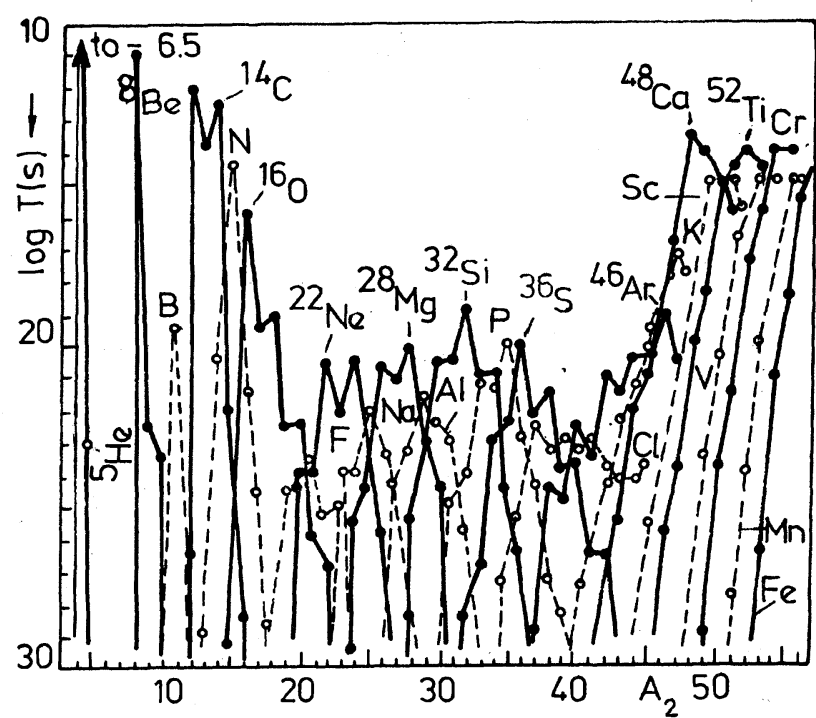

FIG. 5. The decimal logarithm of the lifetimes for the same processes as in Fig. 4.

sequently, we cut Fig. 5 at $T=10^{30} \mathrm{~s}$. The time scale of all figures of this paper (except for Figs. 2 and 8 ) is reversed, because we want to get the impression of the intensity which is proportional to $T^{-1}$.

The most important shell effect is in the region of the double magic ${ }_{82}^{208} \mathrm{~Pb}$ daughter. Similar predictions ${ }^{2}$ for lighter parent nuclei leading to the double magic ${ }_{50}^{132} \mathrm{Sn}_{82}$ show longer lifetimes.

In Fig. 5 one can also see a pairing effect: for odd $N_{2}$ or $Z_{2}$ emitted $\mathrm{HI}$, the lifetime is longer than for their even neighbors. There are few exceptions to this rule concerning $Z_{2}$, as for example ${ }_{7}^{15} \mathrm{~N}_{8}$ and ${ }_{15}^{35} \mathrm{P}_{20}$, but in these cases the neutron numbers $N_{2}$ are magic ones, and proton numbers are almost magic.

For each $A_{2}$ in Fig. 5 one has a $Z_{2}$ giving the minimum lifetime. When $A_{2}$ is increased beyond $A_{2}=16$, the better emitted $\mathrm{HI}$ for a given $A_{2}$ becomes more and more neutron rich and the corresponding parent becomes more neutron deficient nuclei. For $Z_{2} \geq 17$ there is a cut in the curves at higher values of $A_{2}$, due to the fact that the corresponding masses (on the neutron rich side of the emitted particles or the neutron deficient one of the parents) are not available.

The list of all new decay modes given in this figure is very large: ${ }^{5} \mathrm{He},{ }^{8-10} \mathrm{Be},{ }^{11,12} \mathrm{~B},{ }^{12}-16 \mathrm{C},{ }^{13-17} \mathrm{~N},{ }^{15-22} \mathrm{O}$, ${ }^{18-23} \mathrm{~F},{ }^{20-26} \mathrm{Ne},{ }^{23-28} \mathrm{Na},{ }^{23-}{ }^{30} \mathrm{Mg},{ }^{27-32} \mathrm{Al},{ }^{28-36} \mathrm{Si}$, $31-39 \mathrm{P}, \quad 32-42 \mathrm{~S}, \quad 35-45 \cdots \mathrm{Cl}, \quad 37-47 \cdots \mathrm{Ar}, \quad 40-49 \cdots \mathrm{K}$,

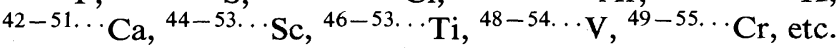
There are more than 140 kinds of exotic radioactivities with $Z_{2}=2-24$ and they continue for $Z_{2}>24$. From these one can select a list of the most intensive processes for a given $A_{2}:{ }^{5} \mathrm{He},{ }^{8} \mathrm{Be},{ }^{11} \mathrm{~B},{ }^{12-14} \mathrm{C},{ }^{15} \mathrm{~N},{ }^{16-20} \mathrm{O},{ }^{21} \mathrm{~F}$, ${ }^{22-24} \mathrm{Ne},{ }^{25} \mathrm{Na},{ }^{26-28} \mathrm{Mg},{ }^{29} \mathrm{Al},{ }^{30-34} \mathrm{Si},{ }^{35} \mathrm{P},{ }^{36-38} \mathrm{~S},{ }^{39,41} \mathrm{Cl}$, ${ }^{40,42-44} \mathrm{Ar},{ }^{45} \mathrm{~K},{ }^{46-50} \mathrm{Ca},{ }^{51-53} \mathrm{Ti},{ }^{54,55} \mathrm{Cr}$, etc. For each element, one or two isotopes are the best emitted: ${ }^{8} \mathrm{Be}$, ${ }^{11} \mathrm{~B},{ }^{12,14} \mathrm{C},{ }^{15} \mathrm{~N},{ }^{16} \mathrm{O},{ }^{21} \mathrm{~F},{ }^{22,24} \mathrm{Ne},{ }^{25} \mathrm{Na},{ }^{26,28} \mathrm{Mg},{ }^{29} \mathrm{Al},{ }^{32} \mathrm{Si}$, ${ }^{35} \mathrm{P},{ }^{36} \mathrm{~S},{ }^{37} \mathrm{Cl},{ }^{46} \mathrm{Ar},{ }^{47} \mathrm{~K},{ }^{48} \mathrm{Ca},{ }^{49} \mathrm{Sc},{ }^{52} \mathrm{Ti},{ }^{53,54} \mathrm{~V},{ }^{54,55} \mathrm{Cr}$, etc.
A detailed illustration of the $N_{1}=126$ neutron and $Z_{1}=82$ proton shell effects in the daughter nucleus is given in Fig. 6, where the lifetime for the 12 decay modes $\left({ }^{8} \mathrm{Be},{ }^{12-14} \mathrm{C},{ }^{15} \mathrm{~N},{ }^{23} \mathrm{~F},{ }^{24,25} \mathrm{Ne},{ }^{28} \mathrm{Mg},{ }^{32} \mathrm{Si},{ }^{46} \mathrm{Ar}\right.$, and $\left.{ }^{48} \mathrm{Ca}\right)$ are plotted versus the daughter neutron number $N_{1}$ for various daughter proton numbers $Z_{1}=80-87$. The minimum value of the lifetime for a given $Z_{1}$ is almost always obtained when $N_{1}=126$. But for some light emitted HI's (like ${ }^{8} \mathrm{Be}$, and ${ }^{12} \mathrm{C}$ ), $Z_{1}$ for the minimum lifetime is not 82 as usual. Nevertheless, the improvement with respect to the values given in Fig. 5 is not larger than one order of magnitude. The even-odd effect can also be better seen in Figs. 6(c), (e), (f), and (h). For even-odd emitted HI's $\left({ }_{6}^{13} \mathrm{C}_{7}\right.$ the $\left.{ }_{10}^{25} \mathrm{Ne}_{15}\right)$ the even $N_{1}$ (odd $N$ ) are preferred. For odd-even $\left({ }_{7}^{15} \mathrm{~N}_{8}\right.$ and $\left.{ }_{9}^{23} \mathrm{~F}_{14}\right)$ the even $Z_{1}$ (odd $Z$ ) are the best.

In Fig. 7, we compare the results obtained using the numerical method (the points) with the analytical formula (the curves). One can see that not far from the magic neutron number of the daughter the agreement is good, but in general the results of the analytic formula are more optimistic than those of the numerical method. This fact may be due to the inclusion of shell effects in the parame-

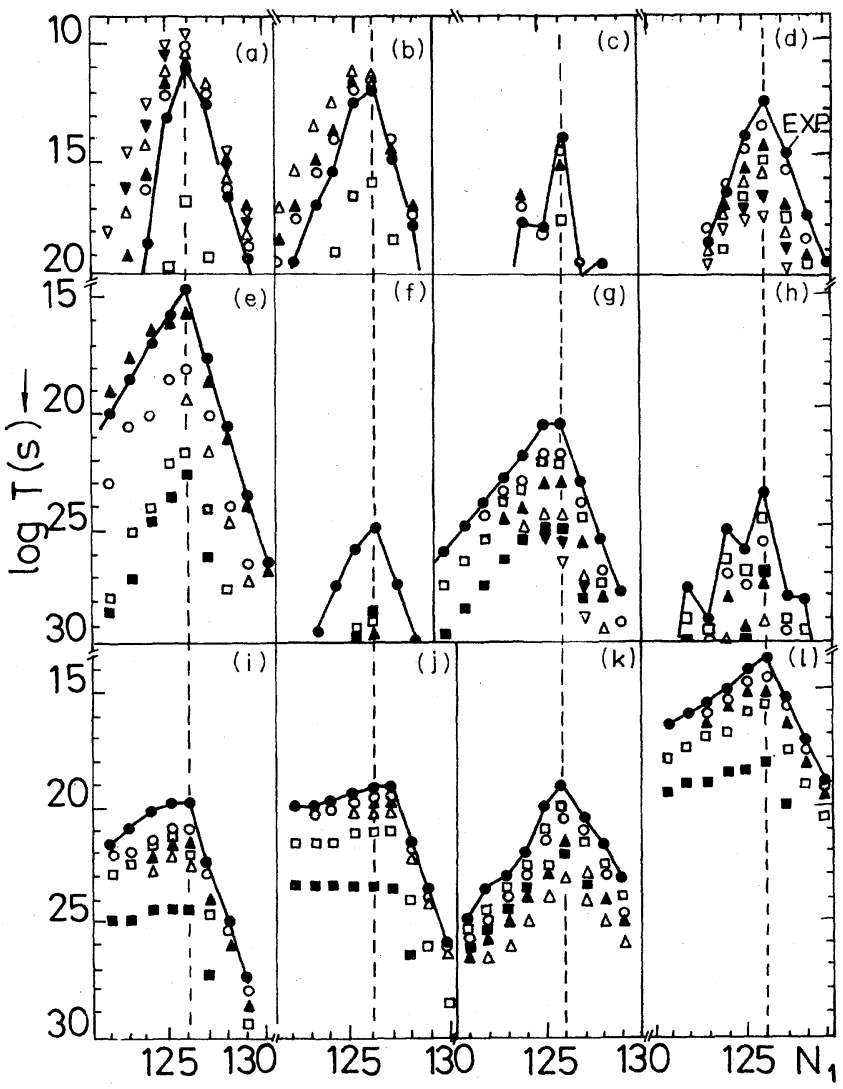

FIG. 6. Lifetimes versus daughter neutron number $N_{1}$ for various decay modes by spontaneous emission of the following nuclei: ${ }^{8} \mathrm{Be}(\mathrm{a}),{ }^{12} \mathrm{C}(\mathrm{b}),{ }^{13} \mathrm{C}(\mathrm{c}),{ }^{14} \mathrm{C}(\mathrm{d}),{ }^{15} \mathrm{~N}(\mathrm{e}),{ }^{23} \mathrm{~F}(\mathrm{f}),{ }^{24} \mathrm{Ne}(\mathrm{g})$, ${ }^{25} \mathrm{Ne}(\mathrm{h}),{ }^{28} \mathrm{Mg}$ (i), ${ }^{32} \mathrm{Si}(\mathrm{j}),{ }^{46} \mathrm{Ar}(\mathrm{k})$, and ${ }^{48} \mathrm{Ca}$ (1). The daughter atomic numbers are the following: $\square-80 ; \square-81 ; 0-82$; $\square-$ $83 ; \Delta-84 ; \triangle-85 ; \nabla-86$, and $\nabla-87$. 


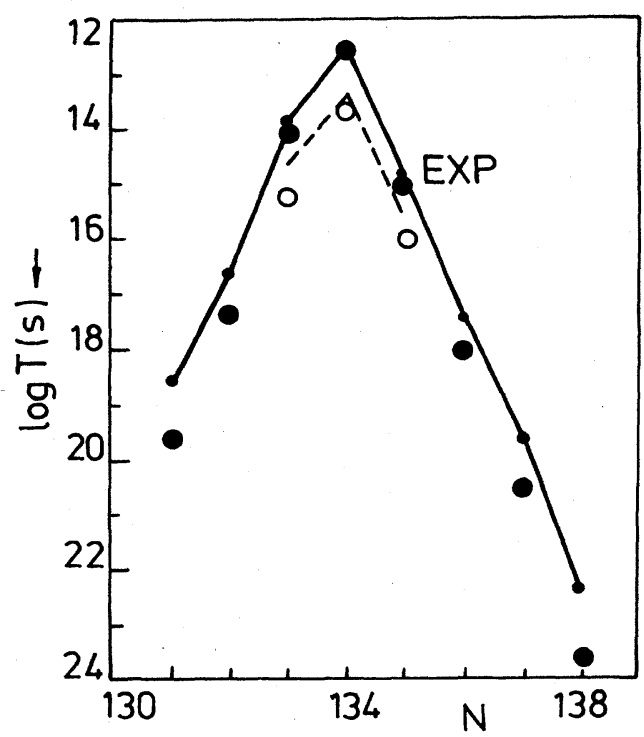

FIG. 7. Comparison of lifetimes for ${ }^{14} \mathrm{C}$ emission from various isotopes of $\mathrm{Ra}$ and $\mathrm{Ac}$, calculated with a numerical method (- Ra, O-Ac) and the analytical formula (-, Ra, $-\ldots$, Ac).

ter $E_{v}$ of the lifetime analytical formula; in the numerical method we have been working with $E_{v}=$ const.

The minimum value obtained for ${ }^{8} \mathrm{Be}$ emission is about 17 orders of magnitude longer than that of the best value for alpha emission. But ${ }^{8} \mathrm{Be}$ itself is an unstable nucleus fissioning into two alpha particles, hence it will be difficult to reject the background in order to detect the ${ }^{8} \mathrm{Be} \alpha$ particles. Nevertheless, in some plastic detectors, ${ }^{45}$ ${ }^{8} \mathrm{Be}$ is very easily identified due to the specific $T$-shaped track.

From Fig. 8, one can see that the lifetime for various $\alpha$ decay modes can be substantially diminished by exciting the emission level above the ground state. A precursor nucleus far off the $\beta$-stability line could populate the emitter excited levels. The $\beta$-delayed HI emission can be analyzed in the same way as the $\beta$-delayed ${ }^{5} \mathrm{He}$ radioactivity. ${ }^{5}$ In fact, we can say that the right word is not $\beta$ delayed but $\beta$-enhanced $\mathrm{HI}$ emission, because the lifetime for HI emission from an excited state is usually longer than that of the $\beta$ decay of the precursor. Alternatively, the feeding of excited levels could be supplied by thermal neutron capture like in $(n, \alpha)$ reactions.

\section{BRANCHING RATIO}

The most important competitor of these new decay modes is usually the alpha decay. Consequently, for some experiments, ${ }^{11,12}$ the absolute value of the lifetime $T$ is not so important as the branching ratio relative to alpha decay $T / T_{\alpha}$. We have shown ${ }^{7,8}$ that from this point of view Rose and Jones ${ }^{11}$ discovered the best emitter of ${ }^{14} \mathrm{C}$-namely ${ }^{223} \mathrm{Ra}$ with a branching ratio

$$
\frac{\Gamma}{\Gamma_{\alpha}}=T_{\alpha} / T=(8.5 \pm 2.5) \times 10^{-10},
$$

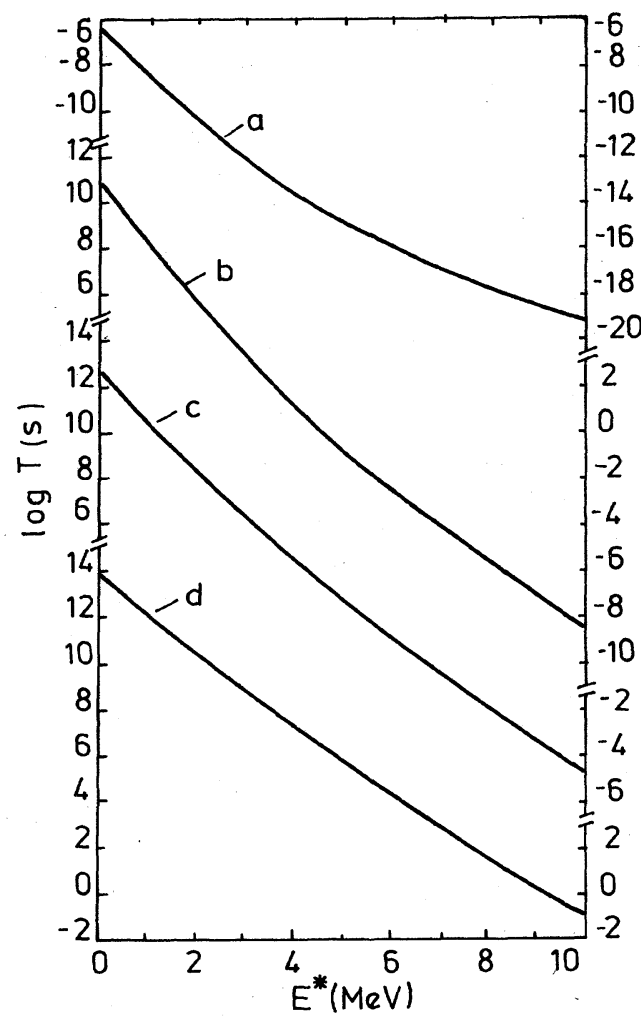

FIG. 8. Lifetimes for the emission from excited levels of ${ }^{4} \mathrm{He}$ (a), ${ }^{8} \mathrm{Be}(\mathrm{b}),{ }^{14} \mathrm{C}(\mathrm{c})$, and ${ }^{48} \mathrm{Ca}(\mathrm{d})$, when the daughter is ${ }^{208} \mathrm{~Pb} . E^{*}$ is the fraction of the excitation energy concentrated in the separation degree of freedom.

confirmed by Alexandrov et al.,$^{12}(7.6 \pm 3.0) \times 10^{-10}$, and by Gales et al. ${ }^{9}(5.5 \pm 2.0) \times 10^{-10}$. Of course, if the $\alpha$ particles could be discriminated (for example deflected ${ }^{9}$ by the magnetic field), the most important quantity is the lifetime. From this point of view, the best ${ }^{14} \mathrm{C}$ emitter is ${ }^{222} \mathrm{Ra}$ rather than ${ }^{223} \mathrm{Ra}$.

The branching ratio relative to the $\alpha$ decay was plotted in Fig. 9 for the same processes as in Fig. 5, leading to the double magic daughter ${ }^{208} \mathrm{~Pb}$. If a measured $\alpha$-decay lifetime is not available in the tables, ${ }^{40,41}$ we calculate $T_{\alpha}$ by using the semiempirical formula. ${ }^{42,41}$

One can see that Fig. 9 is quite different from Fig. 5.

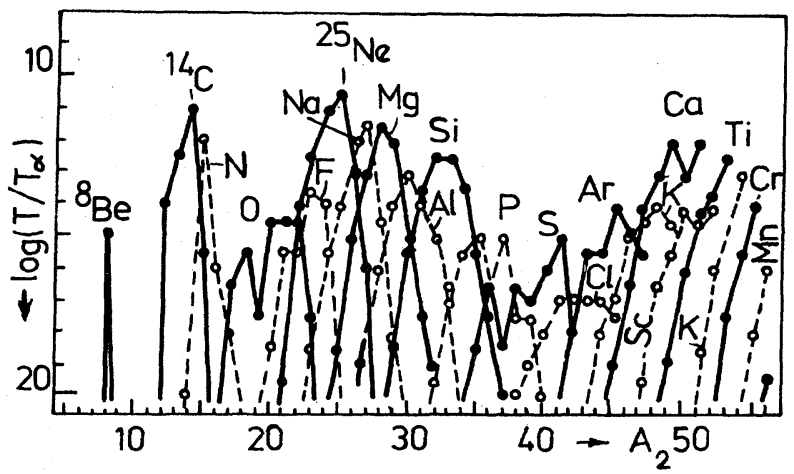

FIG. 9. The branching ratios relative to alpha decay for the same processes as in Fig. 4. 
For example, ${ }^{8} \mathrm{Be}$, which was the best in Fig. $5\left(T \simeq 10^{11}\right)$ is now pushed down three orders of magnitude under $T / T_{\alpha} \leq 10^{12}$ while above this level one has not only ${ }^{14} \mathrm{C}$ ( $T \simeq 12.6)$ and ${ }^{48} \mathrm{Ca}(T \simeq 13.5)$ but also ${ }^{24,25} \mathrm{Ne}$ and ${ }^{28,29} \mathrm{Mg}$ with $T>10^{20}$ s.

We have shown ${ }^{7,8}$ in the example of ${ }^{14} \mathrm{C}$ radioactivity that the simple rule that the optimum case is obtained for the double magic daughter or not too far from it, which works quite well for the absolute value of $\log T$, is no longer useful for the branching ratio $T / T_{\alpha}$. Now we present much more decay modes in Fig. 10.

Only in a small number of cases $\left({ }^{13} \mathrm{C},{ }^{15} \mathrm{~N},{ }^{25} \mathrm{Ne}\right.$, and ${ }^{28} \mathrm{Mg}$ ) the best branching ratio is obtained at $N_{1}=126$. Usually $N_{1}=127$ is preferred for HI's lighter than ${ }^{25} \mathrm{Ne}$ and $N_{1}=125$ for emitted ions heavier than ${ }^{28} \mathrm{Mg}$. $Z_{1}=82$ is on the top for ${ }^{13,14} \mathrm{C},{ }^{15} \mathrm{~N},{ }^{23} \mathrm{~F}$, and ${ }^{48} \mathrm{Ca}$, but we can have $Z_{1}=85$ for ${ }^{8} \mathrm{Be}, Z_{1}=80$ for ${ }^{46} \mathrm{Ar}$, and $Z_{1}=81$ for ${ }^{24,25} \mathrm{Ne},{ }^{28} \mathrm{Mg}$, and ${ }^{32} \mathrm{Si}$. The calculated numbers seem to indicate that it would be difficult to perform experiments due to the weak intensity of these processes, but we know that small branching ratios, relative to $\alpha$ decay, in the range of $10^{-13}-10^{-10}$ have been determined for the spontaneous fission of some Th, U, Np, and Am isotopes.

In the experimental search for new decay modes one can use either Figs. 5 and 6 for the absolute value of $T$, or Fig. 10 for the branching ratio. ${ }^{46}$ Further details about some cases in which a good $T / T_{\alpha}$ ratio is obtained are given in Table $I$. One has to stress that $T_{\alpha}$ is the partial lifetime only for the strongest alpha transition, hence it is sometimes different from the lifetime for all alpha transitions.

In order to see if other decay modes are in competition with $\alpha$ decay, not only $T_{\alpha}$ but also the total half-life $T_{t}$ are given in this table. One can see, for example, that the following nuclei ${ }_{89}^{224} \mathrm{Ac},{ }_{93}^{236} \mathrm{~Np},{ }_{96}^{239} \mathrm{Cm}$, and ${ }_{100}^{253} \mathrm{Fm}$ have $\left|T_{\alpha}-T_{t}\right|$ larger than one order of magnitude. There are

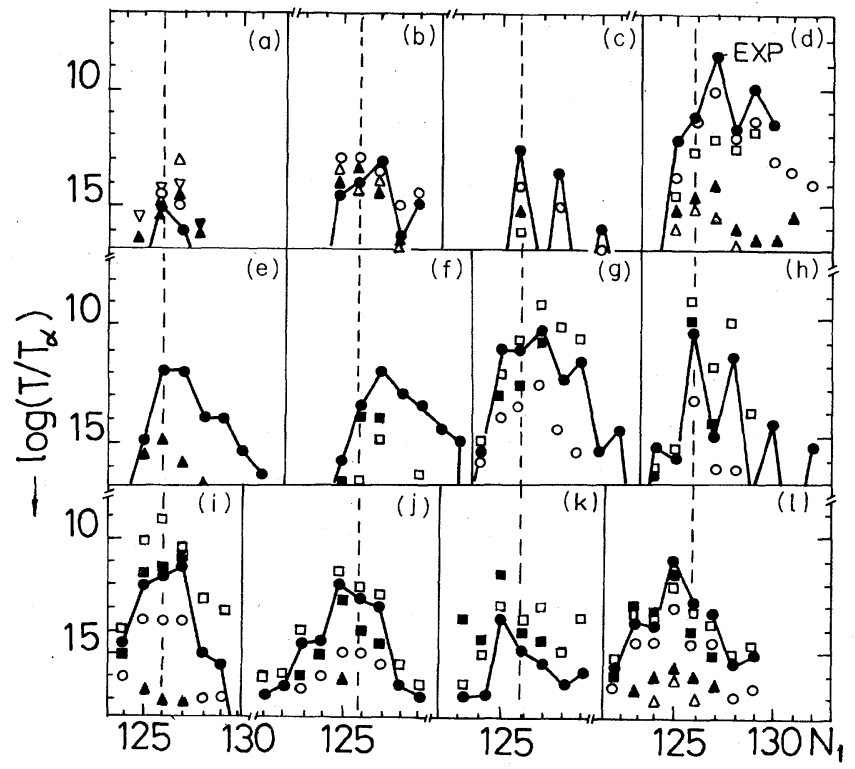

FIG. 10. The branching ratios relative to alpha decay for the same processes as in Fig. 6.

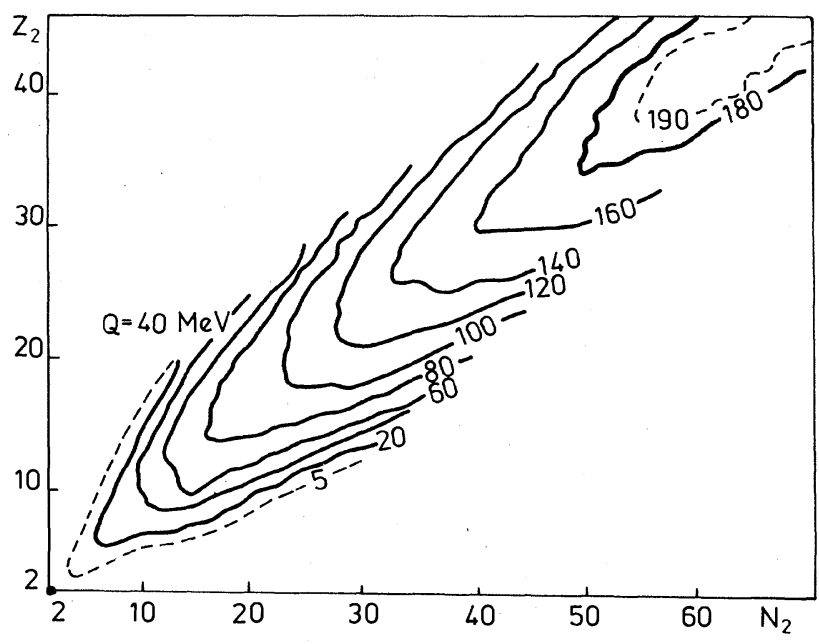

FIG. 11. $Q$ values for the emission of HI's with $Z_{2}$ protons and $N_{2}$ neutrons from a source of ${ }^{233} \mathrm{U}$.

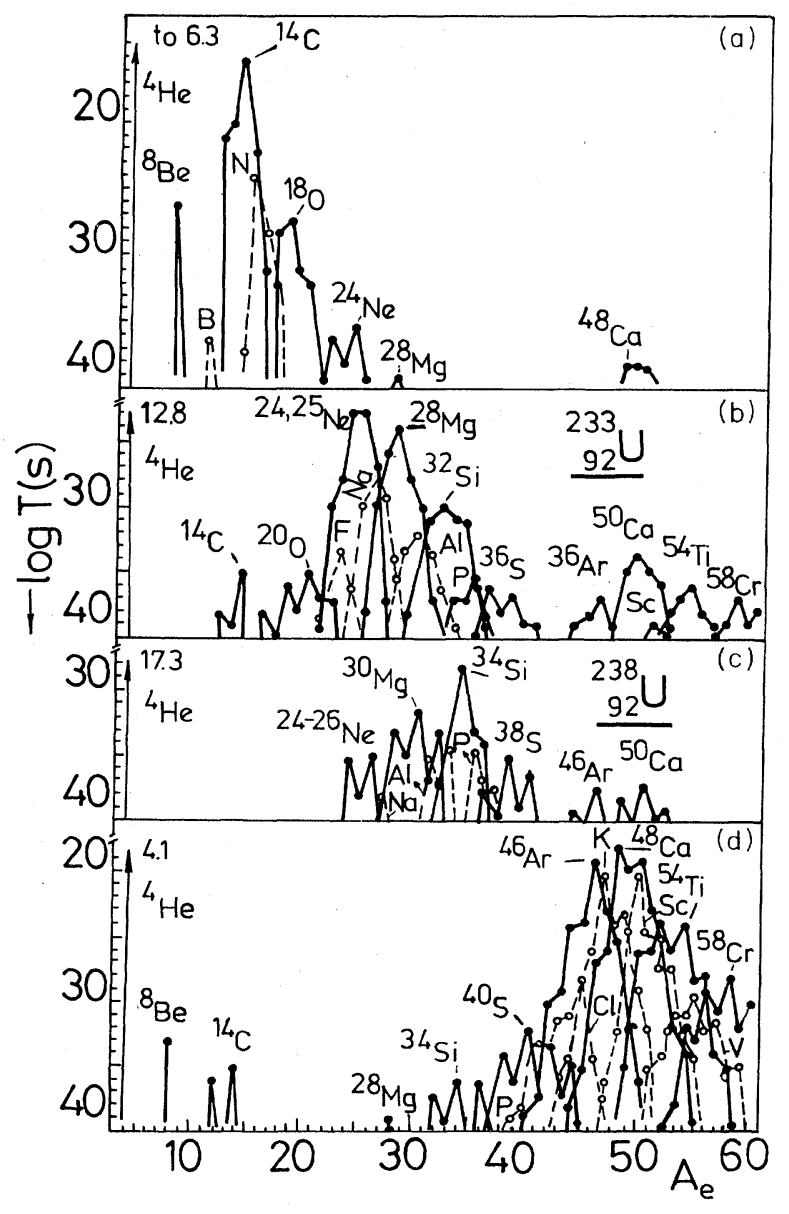

FIG. 12. Lifetime spectra for various modes of decay by HI emission of ${ }^{223} \mathrm{Ra}(\mathrm{a}),{ }^{233} \mathrm{U}$ (b), ${ }^{238} \mathrm{U}$ (c), and ${ }^{254} \mathrm{Fm}(\mathrm{d})$. 
TABLE I. Some high branching ratio (small $T / T_{\alpha}$ ) HI emissions.

\begin{tabular}{|c|c|c|c|c|c|c|c|c|c|c|}
\hline \multirow{2}{*}{$\begin{array}{c}\text { Emitted } \\
\text { HI }\end{array}$} & \multicolumn{2}{|c|}{ Parent } & \multicolumn{2}{|c|}{ Daughter } & \multirow{2}{*}{$\begin{array}{c}E_{k}^{\mathrm{a}} \\
(\mathrm{MeV})\end{array}$} & \multirow{2}{*}{$\begin{array}{c}\log T_{t} \\
\quad(\mathbf{s})\end{array}$} & \multirow{2}{*}{$\begin{array}{l}\log T_{\alpha} \\
\quad(\mathrm{s})\end{array}$} & \multirow{2}{*}{$\begin{array}{c}\log T \\
(\mathrm{~s})\end{array}$} & \multirow[b]{2}{*}{$\log$} & \multirow[b]{2}{*}{$\frac{T}{T_{\alpha}}$} \\
\hline & $Z$ & $A$ & $Z_{1}$ & $N_{1}$ & & & & & & \\
\hline \multirow{7}{*}{${ }_{6}^{14} \mathrm{C}_{8}$} & 87 & 222 & 81 & 127 & 28.2 & 2.94 & 5.4 & 17.3 & & 11.9 \\
\hline & 88 & 222 & 82 & 126 & 31.0 & 1.59 & 1.6 & 12.6 & & 11.0 \\
\hline & & 223 & 82 & 127 & 29.9 & 5.99 & 6.3 & 15.0 & & 8.7 \\
\hline & & 224 & 82 & 128 & 28.6 & 5.53 & 5.5 & 17.4 & & 11.9 \\
\hline & & 226 & 82 & 130 & 26.5 & 10.73 & 10.7 & 22.4 & & 11.7 \\
\hline & 89 & 223 & 83 & 126 & 31.0 & 2.12 & 2.5 & 13.4 & & 10.9 \\
\hline & & 224 & 83 & 127 & 30.0 & 4.02 & 5.6 & 15.4 & & 9.8 \\
\hline \multirow{2}{*}{${ }_{7}^{15} \mathrm{~N}_{8}$} & 89 & 223 & 82 & 126 & 36.8 & 2.12 & 2.5 & 14.7 & & 12.2 \\
\hline & & 224 & 82 & 127 & 35.2 & 4.02 & 5.6 & 17.7 & & 12.1 \\
\hline \multirow{4}{*}{${ }_{10}^{24} \mathrm{Ne}_{14}$} & 91 & 231 & 81 & 126 & 54.1 & 12.00 & 12.0 & 22.0 & & 10.0 \\
\hline & 92 & 232 & 82 & 126 & 55.9 & 9.36 & 9.5 & 20.4 & & 10.9 \\
\hline & & 233 & 82 & 127 & 54.3 & 12.70 & 12.8 & 23.1 & & 10.3 \\
\hline & & 235 & 82 & 129 & 51.5 & 16.33 & 16.6 & 28.1 & & 11.5 \\
\hline \multirow{2}{*}{${ }_{10}^{25} \mathrm{Ne}_{15}$} & 92 & 233 & 82 & 126 & 54.3 & 12.70 & 12.8 & 23.3 & & 10.5 \\
\hline & & 235 & 82 & 128 & 51.7 & 16.33 & 16.6 & 28.1 & & 11.5 \\
\hline \multirow{3}{*}{${ }_{12}^{28} \mathrm{Mg}_{16}$} & 92 & 233 & 80 & 125 & 65.3 & 12.70 & 12.8 & 24.5 & & 11.7 \\
\hline & 93 & 236 & 81 & 127 & 66.2 & 12.56 & 14.0 & 24.5 & & 10.5 \\
\hline & 94 & 236 & 82 & 126 & 70.2 & 7.96 & 8.1 & 19.8 & & 11.7 \\
\hline \multirow{2}{*}{${ }_{12}^{29} \mathrm{Mg}_{17}$} & 92 & 235 & 80 & 126 & 63.7 & 16.33 & 16.6 & 27.4 & & 10.8 \\
\hline & 93 & 236 & 81 & 126 & 66.0 & 12.56 & 14.0 & 25.1 & & 11.1 \\
\hline${ }_{14}^{32} \mathrm{Si}_{18}$ & 96 & 239 & 82 & 125 & 84.6 & 4.03 & 7.0 & 18.9 & & 11.9 \\
\hline${ }_{18}^{46} \mathrm{Ar}_{28}$ & 98 & 251 & 80 & 125 & 103.0 & 10.45 & 10.9 & 22.6 & & 11.7 \\
\hline \multirow{3}{*}{${ }_{20}^{48} \mathrm{Ca}_{28}$} & 100 & 253 & 80 & 125 & 118.2 & 5.41 & 6.7 & 18.4 & & 11.7 \\
\hline & 101 & 254 & 81 & 125 & 121.6 & 3.23 & 4.0 & 15.9 & & 11.9 \\
\hline & 102 & 255 & 82 & 125 & 124.7 & 2.30 & 2.8 & 14.1 & & 11.2 \\
\hline \multirow{2}{*}{${ }_{20}^{50} \mathrm{Ca}_{30}$} & 101 & 258 & 81 & 127 & 119.4 & 6.64 & 6.6 & 17.7 & & 11.1 \\
\hline & 102 & 259 & 82 & 127 & 122.0 & 3.54 & 4.1 & 16.4 & & 12.3 \\
\hline
\end{tabular}

${ }^{\mathrm{a}} E_{k}=Q \cdot A_{1} / A$.

other cases with $T / T_{\alpha} \leq 10^{12}$ but $T_{t} \ll<T_{\alpha}$, which are not mentioned in Table I. Some of the parents in this table are not on the neutron-deficient side of the nuclear chart where $T$ is minimum, due to the fact that $T_{\alpha}$ is also small in this region.

Another important problem in planning an experiment is to know the spectrum of the emitted particles from a given source $A$ and $Z$ available. From the $Q^{\prime}$-value variation with the neutron and proton numbers $N_{2}$ and $Z_{2}$ of the emitted HI, as is shown in Fig. 11 for the ${ }^{233} \mathrm{U}$ parent nucleus, one cannot answer the question. There are many emitted particles with positive $Q$ values. The ridge shape appearing in this figure means that for each $A_{2}$ there is usually one combination of $Z_{2}$ and $N_{2}$ giving the maximum $Q$ value and the corresponding $Q$ increases when $A_{2}$ increases.

In Fig. 12 we present the spectrum of the emitted parti- cles from various sources: ${ }^{223} \mathrm{Ra},{ }^{233} \mathrm{U},{ }^{238} \mathrm{U}$, and ${ }^{254} \mathrm{Fm}$. In the first case one can see that the other HI's $\left({ }^{12,13,15} \mathrm{C}\right)$ which are emitted from ${ }^{223} \mathrm{Ra}$ are more than five orders of magnitude weaker than ${ }^{14} \mathrm{C}$, already measured. We expect that from ${ }^{233} \mathrm{U}$, the most probable emission will have ${ }^{24,25} \mathrm{Ne}\left(T \simeq 10^{23} \mathrm{~s}\right.$ and $T / T_{\alpha} \simeq 10^{10.5}$, as is written in Table I). Then ${ }^{28} \mathrm{Mg}$ follows with $T=10^{24.5}$ and $T / T_{\alpha} \simeq 10^{12}$.

${ }^{238} \mathrm{U}$ is predicted to have $T \simeq 10^{28} \mathrm{~s}$ for the emission of ${ }^{34} \mathrm{Si}$ and $T / T_{\alpha} \simeq 10^{11} ;{ }^{30} \mathrm{Mg}$ is four orders of magnitude lower. But the case of ${ }_{14}^{34} \mathrm{Si}_{20}$ needs to be further examined in order to check the validity of the approximation for the mass value of the daughter $\left({ }_{78}^{204} \mathrm{Pt}_{126}\right)$. For this reason, ${ }^{34} \mathrm{Si}$ was not given in Table I and there are many other similar cases. We need better accuracy in mass predictions and more experimental points to get more confidence in our estimations. From ${ }^{254} \mathrm{Fm}$, the most likely emitted ion is 
${ }^{48} \mathrm{Ca}$ with $T \simeq 10^{18}$ s and $T / T_{\alpha} \simeq 10^{14}$.

By comparing the spectra of all sources in Fig. 12 one can see the trend toward heavier emitted particles when the mass number of the parent increases. This trend is similar with the one observed in the light fragment part of the asymmetric fission fragment mass distribution. In our case it is explained by the shell effect at $N_{1}=126$ :

$$
\begin{aligned}
& { }_{88}^{223} \mathrm{Ra}_{135} \rightarrow{ }_{6}^{14} \mathrm{C}_{8}+{ }_{82}^{209} \mathrm{~Pb}_{127} ; \\
& { }_{92}^{233} \mathrm{U}_{141} \rightarrow{ }_{10}^{24,25} \mathrm{Ne}_{14,15}+{ }_{{ }_{82}}^{209,208} \mathrm{~Pb}_{127,126} ; \\
& { }_{92}^{238} \mathrm{U}_{146} \rightarrow{ }_{14}^{34} \mathrm{Si}_{20}+{ }_{78}^{204} \mathrm{Pt}_{126} ;
\end{aligned}
$$

and

$$
{ }_{100}^{254} \mathrm{Fm}_{154} \rightarrow{ }_{20}^{48} \mathrm{Ca}_{28}+{ }_{80}^{206} \mathrm{Hg}_{126} .
$$

Once more, one can see that the neutron shell effects at $N_{1}=126$ are usually much stronger than the proton ones at $Z_{1}=82$.

In fission, for some heavy Fm isotopes the mass distribution is symmetric because the light fragment has approximately a magic neutron number 82 (or not too far from it) like the heavy fragment. But the $\mathrm{HI}$ emission is essentially an asymmetric process because, at least at present, it is hopeless to believe that the superheavy element ${ }^{416} 164$, for which one has symmetric fission in two fragments ${ }^{208} \mathrm{~Pb}$, will ever be available.

\section{CONCLUSIONS}

In the framework of ASAFM one can obtain reliable values for the nuclear lifetimes relative to the new decay modes if the masses of the three partners (parent and two fragments) are well known and a suitable value of the zero point vibration energy is used. By introducing a shell effect in $E_{v}$ one obtains better agreement with experimental data on $380 \alpha$ emitters and the ${ }^{14} \mathrm{C}$ radioactivity of ${ }^{223} \mathrm{Ra}$ is reproduced. We need, of course, more experimental points (new decay modes with emitted ions heavier than ${ }^{14} \mathrm{C}$ ) to further improve the model, because the lifetime is very sensitive to the details of the barrier shape and parameters. Hence, the present calculations should be taken only as orientative rough estimations.

From a systematic search across the nuclear table, we have shown the great complexity and diversity of the new phenomena: there are more than 140 emitted HI's, various isotopes of the elements with $Z_{2}=2-24$, for which the lifetimes are in the range $10^{10}-10^{30} \mathrm{~s}$. For a given de- cay mode $\left(A_{2}, Z_{2}\right)$ the minimum lifetime is obtained when the daughter has a magic number of neutrons $N_{1}=126$ and a magic number of protons $Z_{1}=82$ or not too far from it. In the trans-tin region, where $N_{1}=82$ plays the major role, the lifetimes are much longer. A pairing effect is also present: usually for odd $N_{2}$ or $Z_{2}$ emitted HI's, the lifetime is longer than for their even neighbors. Almost all nuclides with $Z>40$ are metastable with respect to several decay modes but if the lifetime is very long (for example longer than $10^{30} \mathrm{~s}$ ) one can say that from the practical point of view, the corresponding nucleus is stable. When the mass number $A_{2}$ increases, for $Z_{2}>16$ the position of the optimum-optimorum emitted HI moves in the neutron rich side of the nuclear chart and the parent becomes more and more neutron deficient.

According to our calculations, the best value of the branching ratio for ${ }^{14} \mathrm{C}$ emission relative to $\alpha$ decay $\left(\sim 10^{-9}\right)$ is obtained for the parent ${ }^{223} \mathrm{Ra}$ and was already measured. But in this case the absolute value of the lifetime $\left(\sim 10^{15} \mathrm{~s}\right)$ is by two orders of magnitude longer than that of ${ }^{222} \mathrm{Ra}$ for ${ }^{14} \mathrm{C}$ emission and by five orders of magnitude longer than the minimum value for ${ }^{8} \mathrm{Be}$ radioactivity.

Up to now only penetrabilities, which are not measurable quantities, have been used by some experimenters to choose the most likely spontaneously emitted cluster. This method can yield sometimes wrong predictions in comparison with the lifetime calculations.

The simple rule of the best value for the double magic ${ }^{208} \mathrm{~Pb}$ daughter which works with few exceptions for absolute value of the lifetime, is no longer valid for the branching ratio. For the optimum $T / T_{\alpha}$, the daughter neutron number values $N_{1}$ are spread from 125 to 130 and the proton numbers $Z_{1}$ from 80 to 83 as it was shown in Table I, where there are some interesting cases deserving attention.

The increase of the lifetime due to some units of angular momentum carried by the HI's in order to fulfill the spin and parity conservation laws is usually negligible small, except for some very light emitted particles. The lifetime could be substantially diminished if the transition takes place from an excited level populated by $\beta$ decay of a precursor far off the stability line ( $\beta$-enhanced HI emission) or by a thermal neutron capture, like in $(n, \alpha)$ reactions (neutron-induced HI emission). Finally, we can say that the great complexity and diversity of this phenomenon opens a large field of experimental and theoretical investigations. ${ }^{47-54}$
${ }^{1}$ J. H. Hamilton, P. G. Hansen, and E. F. Zganjar, Rep. Prog. Phys. (to be published).

${ }^{2}$ D. N. Poenaru and M. Ivaşcu, Lecture at the International School on Heavy Ion Physics, Poiana Brasov, 1984; Central Institute of Physics, Bucharest, Report NP-39, 1984.

${ }^{3}$ A. Săndulescu, D. N. Poenaru, and W. Greiner, Fiz. Elem. Chastits. At. Yadra 11, 1334 (1980) [Sov. J. Part. Nucl. 11, $528(1980)]$.

${ }^{4}$ D. N. Poenaru and M. Ivaşcu, Central Institute of Physics, Bucharest, Report NP-17, 1980; Proceedings of the Interna- tional School on Heavy Ion Physics, Poiana Brasov, 1980, edited by A. Rădutǎ and G. Stratan, Central Institute of Physics, Bucharest, 1980, p. 743.

${ }^{5}$ D. N. Poenaru and M. Ivaşcu, Central Institute of Physics, Bucharest, Report NP-27, 1983; J. Phys. (Paris) 45, 1099 (1984).

${ }^{6}$ D. N. Poenaru and M. Ivaşcu, Rev. Roum. Phys. 29, 623 (1984).

${ }^{7}$ D. N. Poenaru, M. Ivaşcu, A. Sǎndulescu, and W. Greiner, Izv. Akad. Nauk SSSR, Ser. Fiz. 48, 1897 (1984); J. Phys. G 10, 
L-183 (1984).

${ }^{8}$ D. N. Poenaru, M. Ivaşcu, A. Săndulescu, and W. Greiner, Joint Institute for Nuclear Research, Dubna, Report E4-84446, 1984.

${ }^{9}$ S. Gales, E. Hourani, M. Hussonois, J. P. Schapira, L. Stab, and M. Vergnes, Phys. Rev. Lett. 53, 759 (1984).

${ }^{10} \mathrm{D}$. A. Bromley, in Proceedings of the Fourth International Conference on Clustering Aspects of Nuclear Structure and Nuclear Reactions, Chester, England, 1984, edited by J. S. Lilley and M. A. Nagarajan (Reidel, Dordrecht, 1985), p. 1.

${ }^{11}$ H. J. Rose and G. A. Jones, Nature (London) 307, 245 (1984).

${ }^{12}$ D. V. Alexandrov, A. F. Belyatsky, Yu. A. Gluhov, E. Yu. Nikolsky, B. V. Novatsky, A. A. Ogloblin, and D. N. Stepanov, Pis'ma Zh. Eksp. Teor. Fiz. 40, 152 (1984).

${ }^{13}$ R. Vandenbosch and J. R. Huizenga, Nuclear Fission (Academic, New York, 1973).

14P. Möller and S. G. Nilsson, Phys. Lett. 31B, 283 (1970).

${ }^{15}$ V. V. Pashkevich, Nucl. Phys. A169, 275 (1971).

${ }^{16}$ B. D. Wilkins, E. P. Steinberg, and R. R. Chasman, Phys. Rev. C 14, 1832 (1976).

17J. R. Nix, Annu. Rev. Nucl. Sci. 22, 65 (1972).

${ }^{18}$ V. M. Strutinsky, Nucl. Phys. A95, 420 (1967); A122, 1 (1968).

${ }^{19}$ M. Brack, J. Damgaard, A. S. Jensen, H. C. Pauli, V. M. Strutinsky, and C. Y. Wong, Rev. Mod. Phys. 44, 320 (1972).

${ }^{20}$ H. J. Fink, J. Maruhn, W. Scheid, and W. Greiner, Z. Phys. 268, 321 (1974).

21J. A. Maruhn and W. Greiner, Phys. Rev. C 13, 2404 (1976).

${ }^{22}$ A. Sǎndulescu, H. Lustig, J. Hahn, and W. Greiner, J. Phys. G 4, L279 (1978).

${ }^{23}$ V. K. Rao, V. K. Bhargava, S. G. Marathe, S. M. Sahakundu, and R. H. Jyer, Phys. Rev. C 19, 1372 (1979).

${ }^{24}$ D. N. Poenaru, D. Mazilu, and M. Ivaşcu, J. Phys. G 5, 1093 (1979).

${ }^{25}$ D. N. Poenaru, M. Ivaşcu, and D. Mazilu, Comput. Phys. Commun. 19, 205 (1980).

${ }^{26}$ D. N. Poenaru, M. Ivaşcu, D. Mazilu, and A. Sǎndulescu, Rev. Roum. Phys. 25, 55 (1980).

${ }^{27}$ W. D. Myers and W. J. Swiatecki, Nucl. Phys. 81, 1 (1966); Ark. Fys. 36, 343 (1967).

${ }^{28} \mathrm{H}$. J. Krappe and J. R. Nix, in Proceedings of the Symposium on Physics and Chemistry of Fission (IAEA, Vienna, 1974), Vol. 1, p. 159.

${ }^{29}$ H. J. Krappe, J. R. Nix, and A. J. Sierk, Phys. Rev. C 20, 992 (1979).

${ }^{30}$ D. N. Poenaru, M. Ivaşcu, and A. Săndulescu, J. Phys. (Paris) 40, L-465 (1979); Rev. Roum. Phys. 24, 917 (1979); J. Phys. G 5, L-169 (1979).

${ }^{31}$ D. N. Poenaru and M. Ivaşcu, J. Phys. G 7, 965 (1981); Rev. Roum. Phys. 27, 129 (1982).

${ }^{32} \mathrm{H}$. C. Pauli and T. Ledergerber, in Proceedings of the International Symposium on Physics and Chemistry of Fission (IAEA, Vienna, 1974), Vol. 1, p. 463.

${ }^{33}$ L. G. Moretto, Nucl. Phys. A247, 211 (1975).

${ }^{34}$ W. J. Swiatecki, Lawrence Berkeley Laboratory Report LBL11403, 1983.
${ }^{35}$ L. G. Sobotka, M. L. Padgett, G. J. Wozniak, G. Guarino, A. J. Pacheco, L. G. Moretto, Y. Chan, R. G. Stokstad, J. Tserruya, and S. Wald, Phys. Rev. Lett. 51, 2187 (1983).

${ }^{36}$ A. H. Wapstra and K. Bos, At. Data Nucl. Data Tables 19 , 215 (1977).

${ }^{37}$ A. H. Wapstra and G. Audi, Nucl. Phys. A432, 1 (1985).

${ }^{38}$ D. N. Poenaru and M. Ivaşcu, Comput. Phys. Commun. 16, 85 (1978).

${ }^{39}$ A. Săndulescu, R. Y. Cusson, and W. Greiner, Lett. Nuovo Cimento 36, 321 (1983).

${ }^{40}$ D. N. Poenaru and M. Ivaşcu, Rev. Roum. Phys. 28, 309 (1983).

${ }^{41}$ D. N. Poenaru and M. Ivaşcu, Rev. Roum. Phys. 29, 587 (1984).

${ }^{42}$ D. N. Poenaru, M. Ivaşcu, and D. Mazilu, J. Phys. (Paris) Lett. 41, L-589 (1980); J. Phys. (Paris) 44, 791 (1983); Comput. Phys. Commun. 25, 297 (1982).

${ }^{43}$ J. Jänecke (private communication).

${ }^{44}$ S. Maripuu, At. Data Nucl. Data Tables 17, 411 (1976).

${ }^{45}$ S. P. Tretyakova (private communication).

${ }^{46}$ In Fig. 2 of Ref. 7 and Fig. 55 of Ref. 8 the branching ratio for ${ }^{14} \mathrm{C}$ emission from ${ }^{221,222} \mathrm{Fr}$ was drawn too small; the present Fig. 10(d) contains the correct values. In Table 2 of the Ref. 7 and Table 6 of Ref. 8 there are missing modes ${ }^{\left({ }^{24} \mathrm{Ne}\right.}$ and ${ }^{28} \mathrm{Mg}$ from ${ }^{232} \mathrm{Th},{ }^{24} \mathrm{Ne}$ from ${ }^{231} \mathrm{~Pa},{ }^{28,29} \mathrm{Mg}$ from ${ }^{235} \mathrm{U},{ }^{34} \mathrm{Si}$ from ${ }^{238} \mathrm{U}$, and ${ }^{48} \mathrm{Ca}$ from ${ }^{252} \mathrm{Cf}$ ). Some of these are included in Table I of this paper. A more complete table will be published elsewhere. The quantity plotted in Fig. 7 of Ref. 3 is the natural logarithm of the penetrability divided by 2 . In Refs. 5 and 8 the coefficient for $K_{o v}$ should be 0.2196 instead of 0.1296. In Eq. (4) of Ref. $7 \frac{1}{2} c$ should multiply both terms arc sin not only the first.

${ }^{47}$ After submission of this paper, new experimental discoveries have been reported: ${ }^{14} \mathrm{C}$ emission (Ref. 48 ) from ${ }^{222,224} \mathrm{Ra}$, ${ }^{24} \mathrm{Ne}$ radioactivity (Ref. 49) of ${ }^{232} \mathrm{U}$, and (Ref. 50) of ${ }^{231} \mathrm{~Pa}$. A superasymmetric fission model using the proximity potential was developed (Ref. 51) and three letters (Refs. 52-54) concerning exotic decays have been published.

${ }^{48}$ P. B. Price, J. D. Stevenson, S. W. Barwick, and H. L. Ravn, Phys. Rev. Lett. 54, 297 (1985).

${ }^{49}$ S. W. Barwick, P. B. Price, and J. D. Stevenson, Phys. Rev. Lett. (to be published).

${ }^{50}$ A. Săndulescu, Yu. S. Zamiatnin, I. A. Lebedev, B. F. Miasoedov, S. P. Tretyakova, and D. Haşegan, Joint Institute for Nuclear Research Rapid Communications, Report No. 5, 1984, p. 5.

${ }^{51}$ Shi Yi-Jin and W. J. Swiatecki, Phys. Rev. Lett. 54, 300 (1985).

52W. Greiner, M. Ivaşcu, D. N. Poenaru, and A. Săndulescu, Z. Phys. A 320, 347 (1985).

${ }^{53}$ A. Sǎndulescu, D. N. Poenaru, W. Greiner, and J. H. Hamilton, Phys. Rev. Lett. 54, 490 (1985).

${ }^{54}$ D. N. Poenaru, M. Ivaşcu, A. Săndulescu, and W. Greiner, Joint Institute for Nuclear Research Rapid Communications, Report No. 5, 1984, p. 29. 